\title{
Faithful Representation of Tactile Intensity under Different Contexts Emerges from the Distinct Adaptive Properties of the First Somatosensory Relay Stations
}

\author{
BBoaz Mohar, ${ }^{1}$-Elad Ganmor, ${ }^{2}$ and Ilan Lampl ${ }^{1}$ \\ ${ }^{1}$ Neurobiology department, Weizmann Institute of Science, Rehovot, 7610001 Israel, and ${ }^{2}$ Center for Neural Science, New York University, New York, New \\ York 10003
}

\begin{abstract}
Adaptation allows neurons to respond to a wide range of stimulus intensities. However, it also leads to ambiguity as the representation of the external world depends on the context. We recorded neurons from Wistar rats' brainstem nuclei belonging to two major somatosensory pathways (lemniscal and paralemniscal) and explored the way in which they encode noisy stimuli under different contexts. We found that although their unadapted intensity-response curves are very similar, the adapted curves of the two pathways are distinctively different as they are optimized for encoding different intensity ranges. Lemniscal neurons most faithfully encoded stimuli when the background intensity was high, whereas paralemniscal cells best encoded stimuli under low intensity context. Intracellular recordings indicate that these differences emerge already at the synaptic level. We suggest that the two pathways synergistically improve the ability of this system to encode a wide range of intensities during natural stimulation, potentially reducing the inherent ambiguity of adaptive coding.
\end{abstract}

Key words: adaptation; brainstem trigeminal complex; intensity coding; PrV; somatosensory; SpVi

\section{Introduction}

The intensity of sensory stimuli during natural behavior can vary by several orders of magnitude, yet the dynamic range of neuronal response is far more limited (Simoncelli and Olshausen, 2001; Dahmen et al., 2010). This discrepancy between the range of sensory stimuli and the response range of neurons is solved across all sensory modalities in a similar manner, adaptation, the change in neuronal sensitivity to the stimulus based on its context (Barlow and Levick, 1969; Wark et al., 2007). The ubiquity of adaptation suggests it confers considerable advantages (Adibi et al., 2013; Cohen-Kashi Malina et al., 2013). However, although adaptation may seem like a perfect solution, it alters the neuronal representation of the external world, introducing response ambiguity. The same stimulus may evoke different responses depending on the context, or different stimuli may evoke the same response as context shifts (Fairhall et al., 2001). One possible solution to the ambiguity problem is to stream the information through parallel pathways, each adapts in an optimized way to

Received 0ct. 20, 2014; revised March 8, 2015; accepted March 25, 2015.

Author contributions: B.M. and I.L. designed research; B.M. performed research; B.M., E.G., and I.L. analyzed data; B.M., E.G., and I.L. wrote the paper.

This work was supported by ISF 1160/11, Minverva, DFG-SFB 1089, and the President of Israel Prize of Excellence in Neuroscience. We thank Shirley Mark, Misha Tsodyks, Yonatan Katz, Rea Mitelman, Inbal Meir, and Katayun Cohen-Kashi Malina for their input on the paper.

The authors declare no competing financial interests.

Correspondence should be addressed to Ilan Lampl, 234 Herzl Street, Rehovot 7610001 Israel. E-mail: ilan.lampl@weizmann.ac.il.

DOI:10.1523/JNEUROSCI.4358-14.2015

Copyright $\odot 2015$ the authors $\quad 0270-6474 / 15 / 356997-06 \$ 15.00 / 0$ encode a different range of intensities. In this study, we explored this possibility in an early stage of the somatosensory system.

Similar to other sensory modalities, tactile information ascends to the cortex through parallel pathways (Shapley et al., 1981; Calford and Aitkin, 1983; Chatterjee and Callaway, 2003). Although it was suggested that the two major pathways in the somatosensory system (lemniscal and paralemniscal; Diamond et al., 1992) provide touch and whisking signals (Yu et al., 2006), or tactile versus noxious inputs (Sewards and Sewards, 2002), their combined role in coding tactile inputs remains unclear. We recently showed (Mohar et al., 2013) that neurons in the brainstem principalis nucleus $(\mathrm{PrV})$, the starting point of the lemniscal pathway, adapt less when the intensity of tactile stimulation increased, whereas neurons of the interpolaris nucleus ( $\mathrm{SpVi}$ ), the first station of the paralemniscal pathway, show the opposite behavior, i.e., adapt more with increasing intensity. We hypothesized that these nuclei may increase the ability of the system to encode a wide range of stimulus intensities in different contexts.

\section{Materials and Methods}

Animals. Animal surgeries and in vivo recordings were performed as previously described (Katz et al., 2006). Briefly, recordings were made from young adult (4- to 8-weeks-old) Wistar rats of either sex grouped in up to five animals per cage that had a $12 \mathrm{~h}$ light/dark cycle. After initial anesthesia with ketamine and xylezene (100 and $10 \mathrm{mg} / \mathrm{kg}$ respectively, i.p.; Ketaset, Fort Dodge Animal Health), a tracheotomy was made following local subcutaneous injection of lidocaine. Rats were mounted in a stereotaxic device and respirated with a mixture of isoflurane $(0.5-1.5 \%)$ or Halothane $(0.25-1 \%)$ and oxygen-enriched air. The levels of end-tidal $\mathrm{CO}_{2}$ and heart rate (200-400 beats/min) were monitored throughout the experiment. Body temperature was kept at $37^{\circ} \mathrm{C}$ using a heating 
A

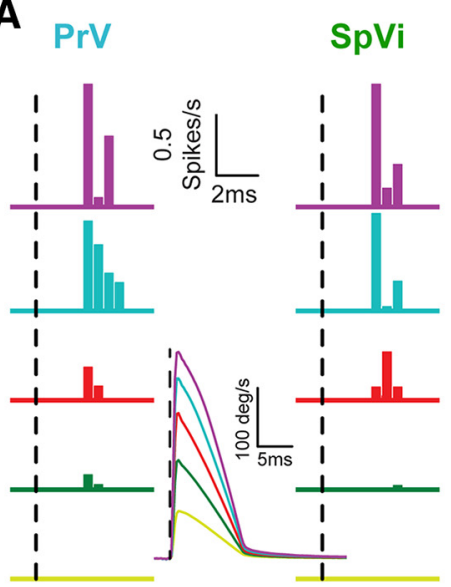

B

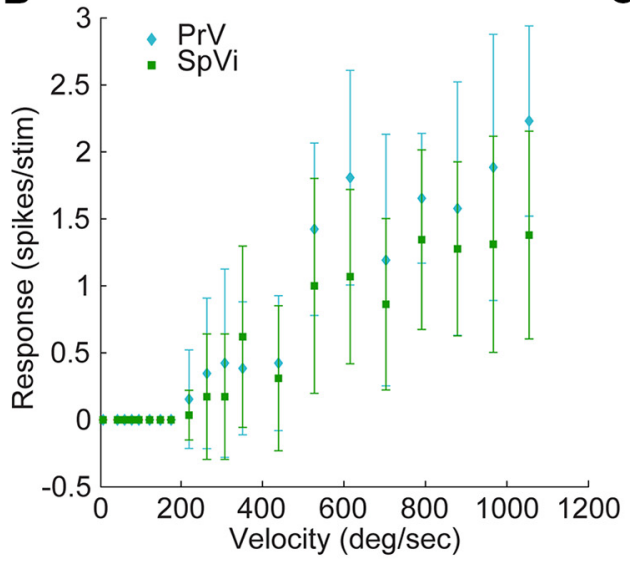

C

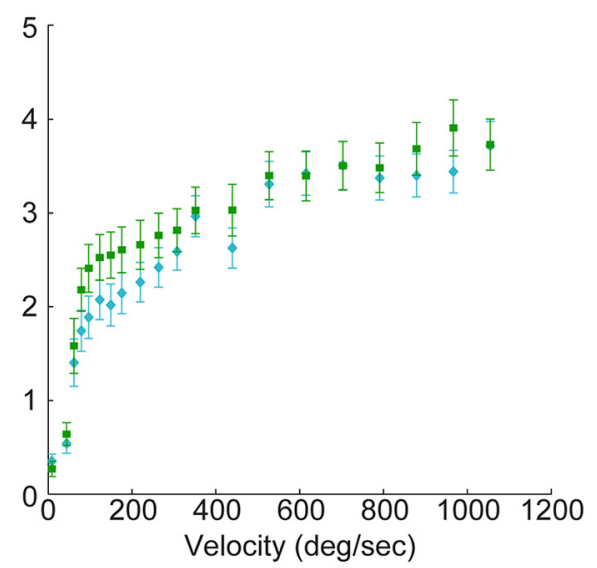

Figure 1. Unadapted responses of the PrV and SpVi are similar. $A$, PSTH examples of responses to single-whisker deflection of different velocities delivered at $0.5 \mathrm{~Hz}$ (inset) from a PrV neuron (left) and a SpVi neuron (right). $\boldsymbol{B}$, Averaged responses of the cells in $\boldsymbol{A}$ as a function of velocity (mean \pm SD). $\boldsymbol{C}$, Population data from 28 PrV neurons and 27 SpVi neuron (mean \pm SE). There are no significant differences in all velocities.

blanket and rectal thermometer (TC-1000; CWE). All surgical and experimental procedures were conducted in accordance with the regulations of the Weizmann Institute Animal Care and Use Committee.

Electrophysiology. After the skin was removed from the top and rostral parts of the head, we exposed the dorsal part of the cerebellum and the first cervical segment of the spinal cord. The dura was removed and the area was continuously washed with ACSF. Extracellular recordings were obtained using $1 \mathrm{M} \Omega$ tungsten microelectrodes (AM-573220; A-M Systems). Intracellular recording were performed using sharp glass electrodes (borosilicate outer diameter: $1.5 \mathrm{~mm}$; inner diameter: $0.86 \mathrm{~mm}$ ) pulled with a P-97 micropipette puller (Sutter Instruments), and filled with $2 \mathrm{M} \mathrm{K}$-acetate (resistance of $60-100 \mathrm{M} \Omega$ ) and $20 \mathrm{~mm}$ QX-314 to prevent firing. The recording site was $2-3 \mathrm{~mm}$ lateral to the midline and $1 \mathrm{~mm}$ dorsal to the meeting point of the brainstem and cerebellum. Using a motorized manipulator (MX7600; Siskiyou) the electrode was inserted with an angle of $2^{\circ}-7^{\circ}$ in relation to the horizontal plane and in parallel to the sagittal plane. The exposed tissue was then covered with warm agar (3\% in $0.9 \%$ saline) to lessen respiratory and cardiovascular movements and prevent the tissue from drying. Extracellular signals were acquired using a model 3000 amplifier (A-M Systems) band passed $0.3-3 \mathrm{kHz}$, amplified $1000-10,000$, and digitized at $10 \mathrm{kHz}$ using homemade acquisition software in LabView (National Instruments). Single units were isolated using WaveClus software (Quiroga et al., 2004) in MATLAB (MathWorks). Intracellular signals were acquired using a Multiclamp$700 \mathrm{~B}$ amplifier (Molecular Devices) low-passed filtered at $3 \mathrm{kHz}$ and then digitized at $20 \mathrm{kHz}$ using the same acquisition hardware and software.

Whisker stimulation and protocols. Whiskers were trimmed to a length of $10-20 \mathrm{~mm}$. The primary whisker was inserted into a $2 \mathrm{~mm}$ plastic cone glued into a metal pipette attached to a galvanometer servo-controlled motor with the matching driver and controller $(6210 \mathrm{H}$ and MicroMax 677xx; Cambridge Technology). The displacement near the tip of the metal pipette was measured off-line using an optical displacement measuring system (optoNCDT 1605; Micro-Epsilon) verifying that there is no ringing. To study the coding of different intensity contexts in the PrV and SpVi, three stimulation sets were used, each with 20 patterns that were $2 \mathrm{~s}$ long. Velocities and intervals of stimulation were randomly distributed with a normal distribution for the high-intensity context set (frequency: $13.4 \mathrm{~Hz} \pm 7.8 \mathrm{~Hz}$; velocity: $504.6 \pm 234.2 \%$ s; ISI range: 21-236 ms) and then their velocities were scaled for the medium $(166.5 \pm 77.3 \%)$ and low $(50.5 \pm 23.4 \%$ s $)$ intensity context sets while keeping their interstimulus intervals constant. All sets were repeated five to six times in all cells. Each whisker deflection was composed of a fastrising voltage command followed by a $20 \mathrm{~ms}$ ramp-down signal. The fast whisker deflection had a constant rise time of $\sim 1 \mathrm{~ms}$; thus, the amplitude and speed of deflection grow together with the magnitude of the voltage command, following a quasilinear relationship. Intertrial intervals between each of the 20 patterns were $3 \mathrm{~s}$ and trials were pseudorandomized. Velocities and frequencies are reported as mean \pm SD. For the unadapted responses, one whisker deflection was given every $2 \mathrm{~s}$ and trials were also pseudorandomized.

Statistical analysis. The statistical analyses of the populations were conducted using two-way ANOVA followed by Bonferroni's multiplecomparisons test. A $p$ value $<0.05$ was considered significant and results are reported as mean \pm SE unless stated otherwise. Normality of the population $R^{2}$ data was tested using Lilliefor's test (linear fit: $D_{\mathrm{f}}=252, k_{\text {stat }}=0.042, p=$ 0.305 ; sigmoid fit: $\left.D_{\mathrm{f}}=252 k_{\text {stat }}=0.53, p=0.4\right)$. A $20 \mathrm{~ms}$ window was used to calculate either spike count or EPSP peak. From which, coefficient of determination $\left(R^{2}\right)$ was calculated after disregarding the first three stimuli in each trial (a window of $320 \pm 60 \mathrm{~ms}$, mean \pm SD) to compute the average response of each cell, using either a linear model $(y=a x+b)$ or a sigmoid one $\left(y=a+\frac{b}{1+e^{-c x+d}}\right)$. Thus, $R^{2} \equiv 1-\frac{S S_{\text {residual }}}{S S_{\text {total }}}$, where: $S S_{\text {residual }}=\Sigma_{i}\left(y_{i}-f_{i}\right)^{2}, S S_{\text {total }}=\Sigma_{i}\left(y_{i}-\bar{y}\right)^{2}$, and $f_{i}$ is the model's prediction to the observation $y_{i}$. Sigmodal fits were constrained to be positive $(a>0)$.

\section{Results}

To better understand how adaptation influences coding of stimulus intensity in the PrV and SpVi, we investigated their unadapted responses to different intensities of brief stimuli, known to evoke profound responses at different stages the somatosensory system (Ganmor et al., 2010). We stimulated the principal whisker of anesthetized rats at a low rate $(0.5 \mathrm{~Hz})$ and recorded the firing response profile from both nuclei (Fig. $1 A, B$ shows example cells) independently. The unadapted responses were similar in both nuclei over a large range of stimulus velocities (Fig. $1 C$; $\operatorname{PrV} n=$ 28 , SpVi $n=27$, two-way ANOVA, Bonferroni's correction, $p>$ 0.05 for all velocities). It should be noted that both curves start to saturate at a similar low intensity. This suggests that in the unadapted state, both pathways encoded velocity in a similar way.

To determine how the two nuclei encode different intensity contexts under adaptation, we formed three sets of stimuli (low, high, and medium intensity contexts), all scaled versions of the same basic 20 patterns of mechanical whisker deflections (see Materials and Methods; Fig. 2A-C, bottom). We found that the two brainstem nuclei are optimized for encoding different intensities. The relationship between stimulus intensity and response 
A

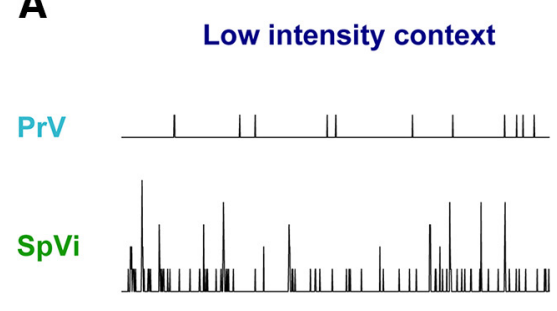

B

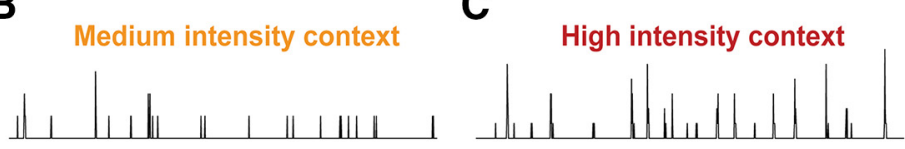

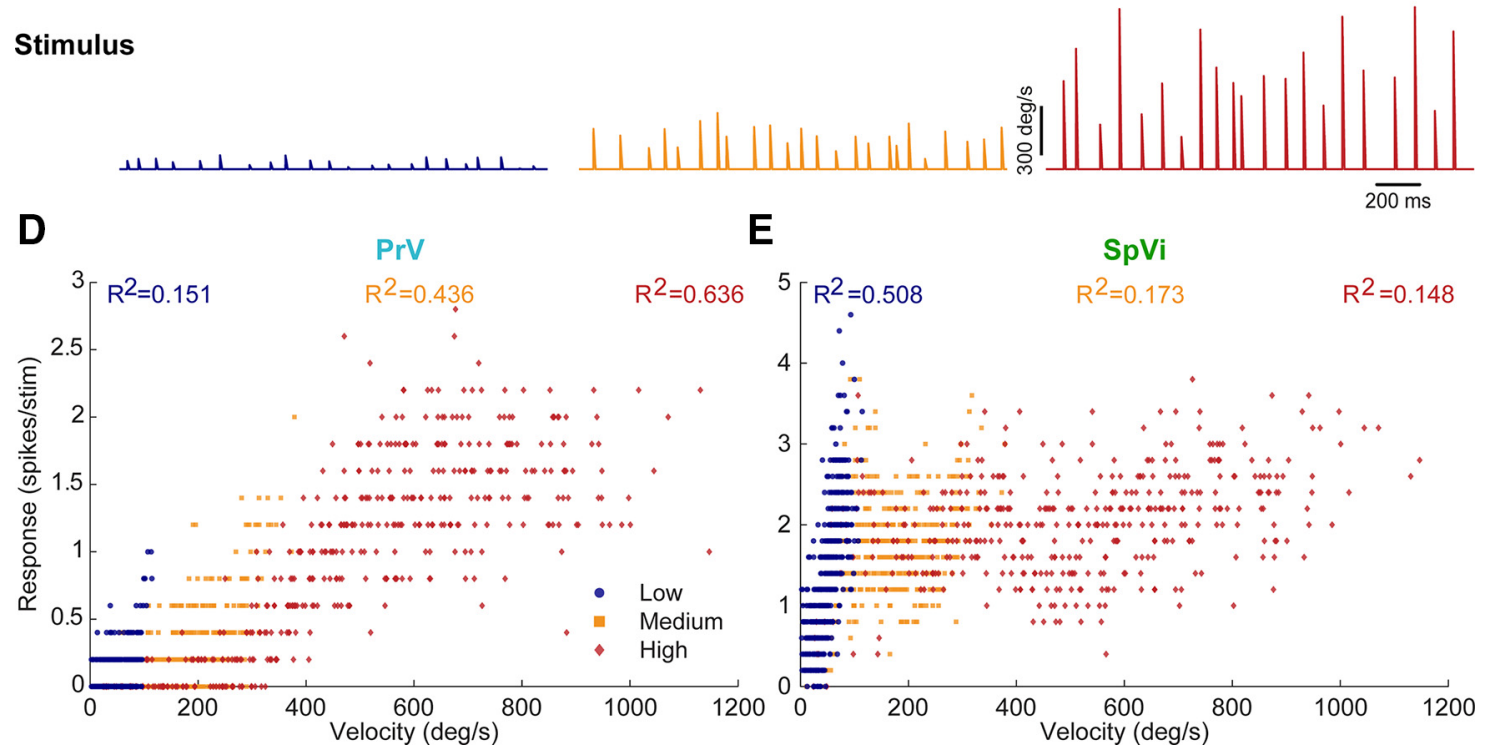

Figure 2. Coding of different contexts in extracellular recording examples of PrV and SpVi neurons. $\boldsymbol{A}-\boldsymbol{C}$, PSTHs from the PrV (average of 5 repeats; top) and SpVi (average of 5 repeats; middle) for three example stimulation patterns (bottom) from the low- $(\boldsymbol{A}$, blue), medium- ( $\boldsymbol{B}$, orange), and high-intensity $(\boldsymbol{C}$, red) contexts. $\boldsymbol{D}, \boldsymbol{E}$, Summary of the responses of the PrV $(\boldsymbol{D})$ and $\operatorname{SpVi}(\boldsymbol{E})$ example neurons in $\boldsymbol{A}-\boldsymbol{C}$. In the PrV encoding of stimulus velocity improves as we increase the mean intensity, whereas in the SpVi the opposite is true. This was quantified by the coefficient of determination of a linear regression $\left(R^{2}\right)$.

(Fig. 2A-C, top and middle) was estimated by calculating the coefficient of determination $\left(R^{2}\right)$ between stimulus velocity (stimulation is described by a quasilinear relation between amplitude and velocity; Ganmor et al., 2010) and the peristimulus time histogram (PSTH), after disregarding the first three stimuli in each trial. In the PrV, increasing the stimulus intensity increased the $R^{2}$ values (Fig. $2 D$ shows an example cell). In the $\mathrm{SpVi}$, on the other hand, $R^{2}$ values decreased as intensity increased (Fig. $2 E$ shows an example cell).

The differences between the two nuclei are observed in extracellularly recorded individual cells across the population. $R^{2}$ increased for most PrV cells (Fig. $3 A$, right; $R^{2}$ increased with intensity for 38/41 cells in low vs high) when the dynamic range was higher and vice versa for SpVi cells (Fig. $3 B$, right; $R^{2}$ decreased with intensity for $37 / 45$ cells in low vs high). In these figures, data were compared across all the combinations of intensity contexts, where the responses to the higher intensity are presented as function of the lower intensity. Hence, points above the diagonal are cells that exhibit better coding in a higher-intensity context, and points below the diagonal constitute the opposite ( $p$ values are reported in each panel and crosshairs are mean \pm SEM). This inverse image of coding emerges across the two nuclei when $R^{2}$ for each context was averaged across cells and compared (Fig. $3 C ; n=41$ for $\operatorname{PrV}, n=45$ for SpVi; two-way ANOVA interaction effect; $\left.F_{(2,252)}=33.02, p=1.86 \mathrm{e}^{-15}\right)$. Whereas for the low intensity, the $R^{2}$ for $\mathrm{SpVi}$ is higher than for the $\mathrm{PrV}$ (SpVi: $0.47 \pm 0.03$, PrV:0.2 $\pm 0.03 ; t_{(252)}=5.928, p=1 \mathrm{e}^{-8}$, Bonferroni's correction), at medium and high-context intensities $R^{2}$ is higher for PrV (medium, SpVi: $0.36 \pm 0.03$, PrV:0.47 \pm 0.04; $t_{(252)}=2.443, p=0.046$; high, SpVi: $0.26 \pm 0.02, \operatorname{PrV}: 0.5 \pm 0.03$; $\left.t_{(252)}=5.077, p=7.5 \mathrm{e}^{-7}\right)$.

To determine whether our assumption of linearity when fitting the data is the cause of the observed differences, we also tested a sigmoidal fit to the responses of each cell. Although a sigmoidal response curve is biologically more plausible, a good fit to a sigmoidal function is not an intuitive correlate to better coding, as a step-like sigmoidal response can only discriminate between two states. Similar to our linear model, $R^{2}$ values of the sigmoidal fit increased with intensity for the $\operatorname{PrV}$ and decreased for the SpVi (Fig. $3 D ; \operatorname{PrV}-n=41 ; \operatorname{SpVi-} n=45$; two-way ANOVA interaction effect, $\left.F_{(2,252)}=13.46, p=2.79 \mathrm{e}^{-6}\right)$. Hence, for $\mathrm{PrV}$ data scattering is reduced with increasing intensity and vice versa for SpVi (low, PrV: $0.73 \pm 0.05, \mathrm{SpVi}$ : $0.92 \pm 0.04$; $t_{(252)}=2.515, p=0.0125$; medium, PrV: $0.95 \pm 0.05$, SpVi: $0.78 \pm 0.04 ; t_{(252)}=2.275, p=0.053$; high, PrV: $0.96 \pm 0.05$, SpVi: $\left.0.61 \pm 0.03 ; t_{(252)}=4.714, p=4.02 \mathrm{e}^{-6}\right)$. Our results indicate that most cells in each nucleus obeyed the same dependency on stimulus intensity, suggesting that a similar picture will emerge in the population average response.

The population average spike-count response curves (Fig. $3 E, F ; \operatorname{PrV}, n=41 ; \mathrm{SpVi}, n=45)$, strongly support our analysis of individual cells, showing even greater $R^{2}$ values and clearer differences between the two nuclei when compared with the mean $R^{2}$ of individual cells. The higher values of $R^{2}$ when spike-count data were pooled, support classical models of population coding (Averbeck et al., 2006). Importantly, because no significant differences across the two populations were found for the unadapted response (the additional units that were presented in Fig. 

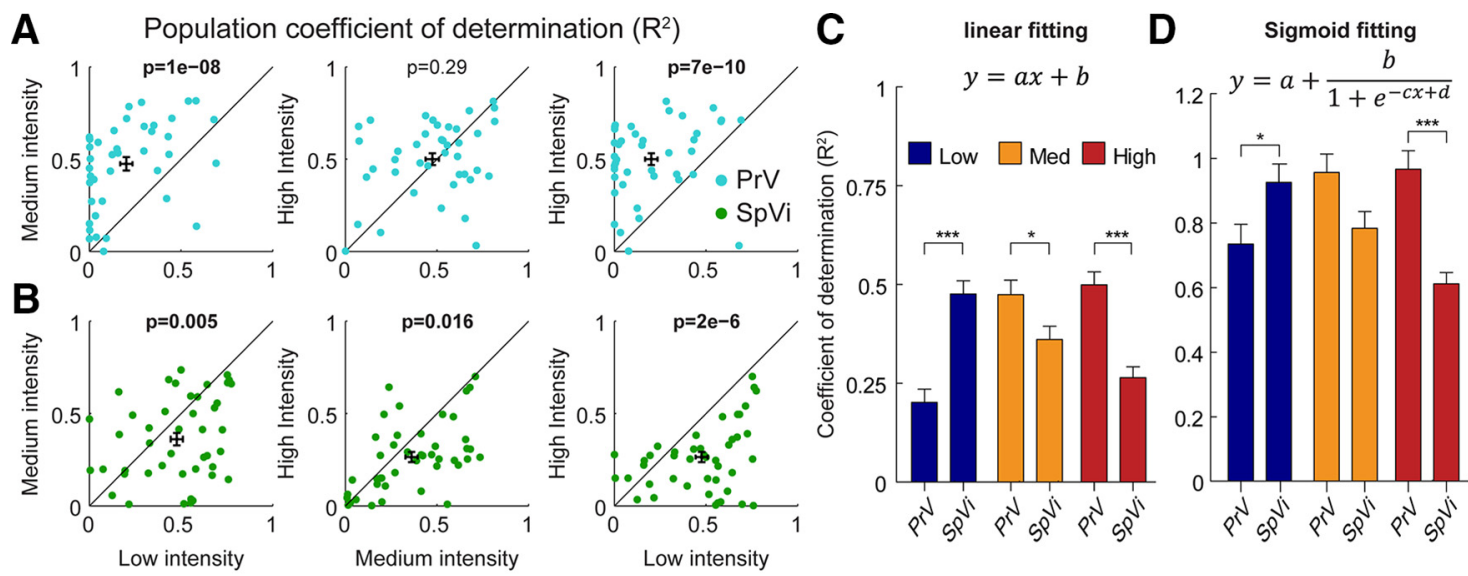

$\mathbf{E}$

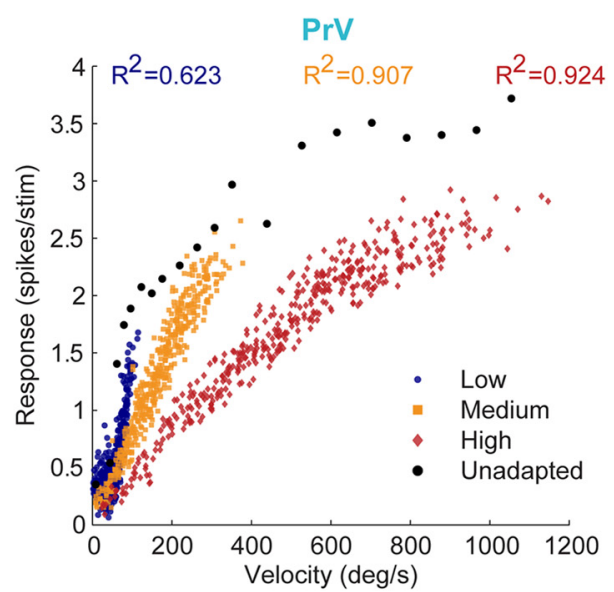

$\mathbf{F}$

$\mathrm{SpVi}$

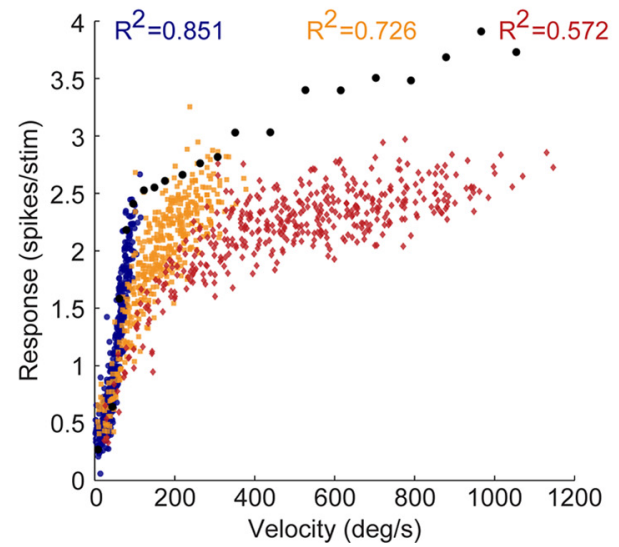

G

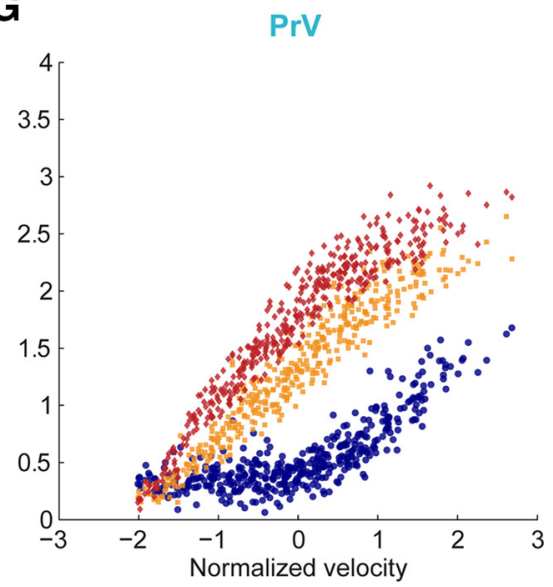

H

$\mathrm{SpVi}$

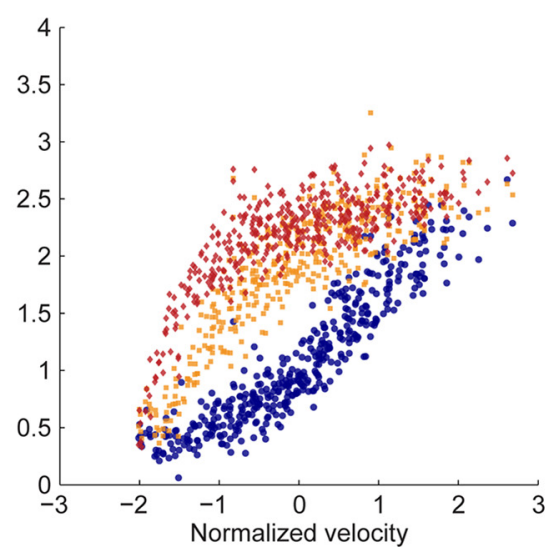

Figure 3. PrV and SpVi representations of intensity are optimized at different contexts. $A, B$, Correlation coefficients for all $\operatorname{PrV}(n=41)$ and SpVi $(n=45)$ neurons with population average and SE as crosshairs. In each panel, the correlation for a higher-stimulus intensity context is presented against a lower-intensity context. $C$, $\boldsymbol{D}$, Comparison of mean correlations between stimulus velocity and response for the PrV and SpVi obtained using linear $(\boldsymbol{C}$ and sigmodal $(\boldsymbol{D})$ fits. $\boldsymbol{E}, \boldsymbol{F}$, Averaged responses of $\operatorname{PrV}(\boldsymbol{E})$ and $\operatorname{SpVi}(\boldsymbol{F})$ neurons as a function of stimulation velocity at the three intensity contexts: low (blue circles), medium (orange squares), and high (red diamonds). Although unadapted responses (black circles) are similar for both nuclei, PrV codes stimulus intensity more linearly at the high-intensity context and the SpVi better codes the low-intensity context. $\boldsymbol{G}, \boldsymbol{H}$, Population responses as in $\boldsymbol{E}, \boldsymbol{F}$ as a function of normalized velocity (Z-scores).

1 are superimposed in Fig. 3E,F, black points), the differences between the two nuclei are a direct consequence of adaptation. For better visualization of the same adapted data, stimulus velocity was normalized for each range by Z-scoring (Fig. 3G,H).

To see whether the differences in coding of stimulus intensity originate in the synaptic input to these cells, we recorded the membrane potential of a subset of cells (Fig. 4A-E shows two example cells) using sharp electrodes. The relationship between stimulus intensity and response was estimated by calculating $R^{2}$ values between stimulus velocity and the peak amplitude of the EPSP. Whereas for $\operatorname{PrV}$ cells $R^{2}$ values increased with velocity, the $\mathrm{SpVi} R^{2}$ values decreased with increasing velocity. Thus indicating that differences between the two nuclei are of subthreshold synaptic origin and not a consequence of the spiking threshold (Fig. $4 F$; $\operatorname{PrV}, n=4$, SpVi, $n=5$; two-way ANOVA interaction effect, $F_{(2,16)}=7.09, p=0.006$; low: $0.36 \pm 0.12$ vs $0.69 \pm 0.1 p=$ 0.035 ; medium: $0.53 \pm 0.09$ vs $0.39 \pm 0.21 p>0.05$; high: $0.84 \pm$ 0.03 vs $0.18 \pm 0.14 p=0.026$ ). 
A

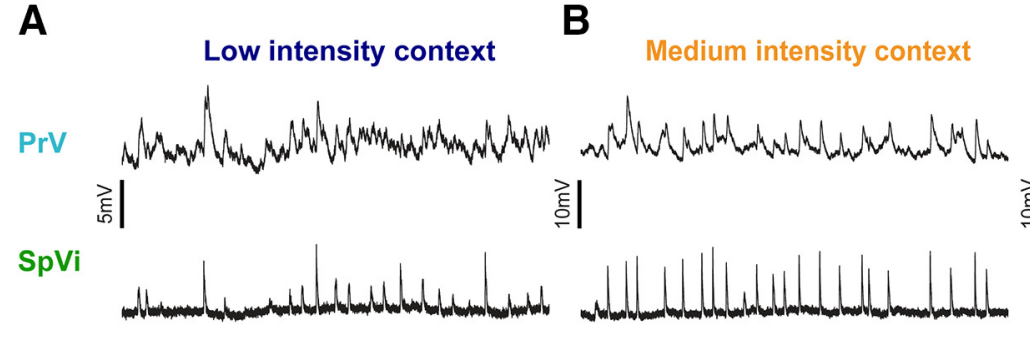

C

High intensity context
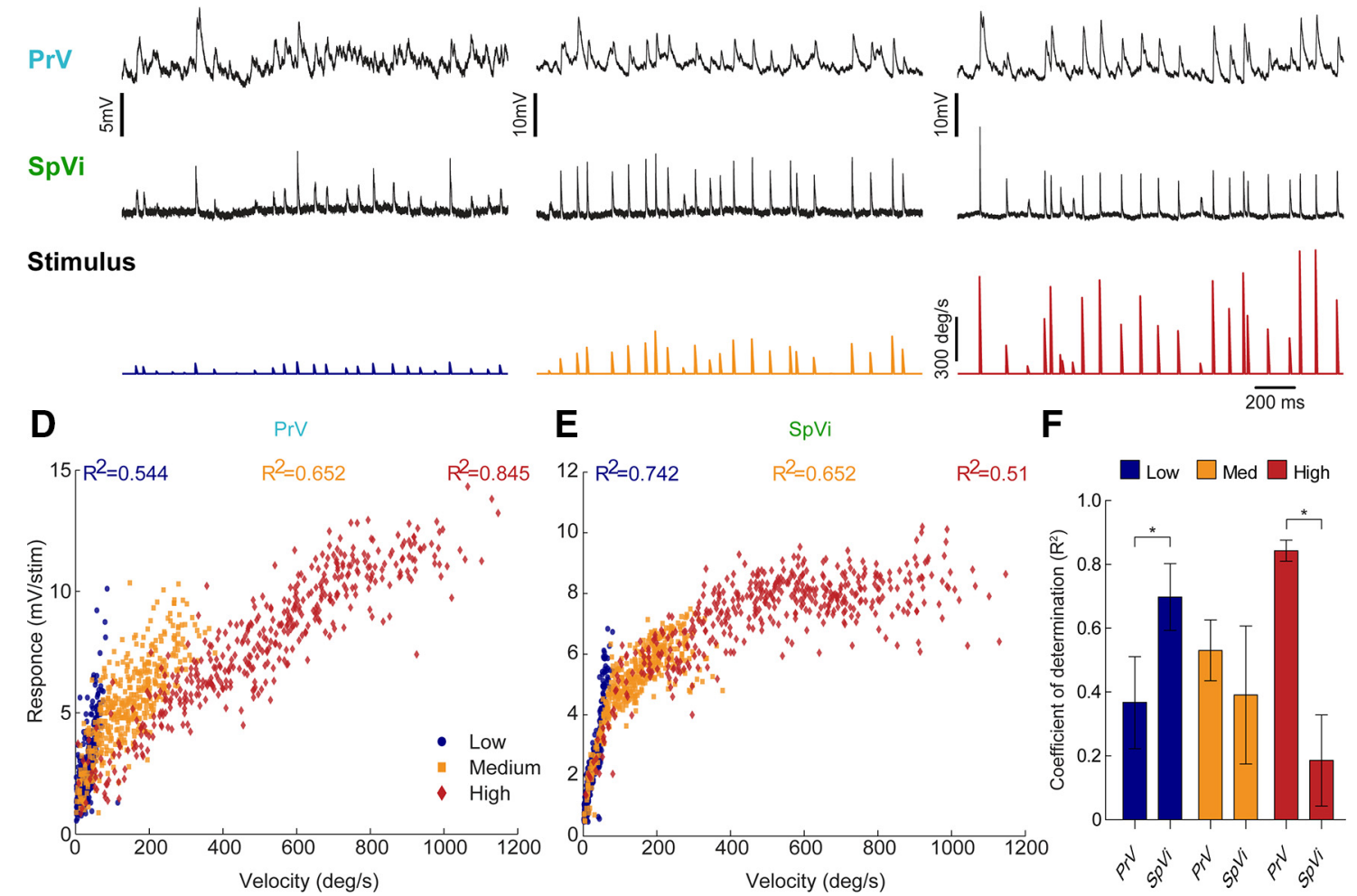

हो)
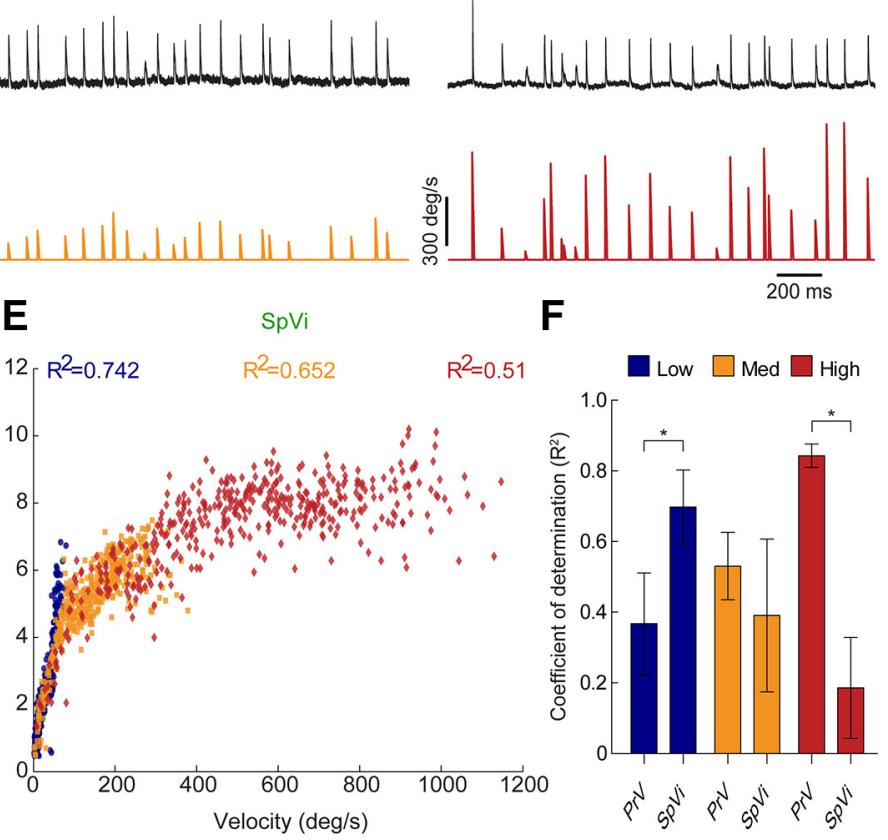

Figure 4. Intracellular coding of PrV and SpVi neurons of different contexts. $A-C$, Average voltage traces from a PrV cell ( $n=5$ repeats; top) and a SpVi cell ( $n=5$ repeats; middle) for the low$(\boldsymbol{A})$, medium- $(\boldsymbol{B})$, and high-intensity $(\boldsymbol{C})$ contexts (stimulation patterns are depicted below the recordings). $\boldsymbol{D}, \boldsymbol{E}$, Summary of the EPSP peak amplitude responses of the PrV (D) and SpVi $(\boldsymbol{E})$ example neurons in $\boldsymbol{A}-\boldsymbol{C}$. In the PrV encoding of stimulus velocity improves as we increase the mean intensity, whereas in the SpVi the opposite is true (quantified by the coefficient of determination of a linear regression between EPSP peak and stimulus velocity). $\boldsymbol{F}$, Population comparison of mean coefficient of determination between stimulus velocity and response for the $\operatorname{PrV}(n=4)$ and SpVi $(n=5)$ obtained using linear fitting.

These data demonstrate that during adaptation PrV neurons are optimized for encoding high-intensity context of stimulation, whereas SpVi neurons better encode the intensity under a lowintensity context. Together with our intracellular recordings, our results suggest that different synaptic adaptation mechanisms are responsible for the unique coding properties of the two nuclei.

\section{Discussion}

In this study, we recorded single units in the brainstem trigeminal complex while applying noisy stimuli of different intensity ranges to the principal whisker. This was done in anesthetized animals to control for brain states (Poulet et al., 2012), corticofugal modulation (Furuta et al., 2010), as well as to avoid active whisking (Zagha et al., 2013). We report that although the two nuclei encode unadapted stimuli in a very similar manner, adaptation introduces distinct differences in their coding behavior. Under adaptation, neurons in the $\operatorname{PrV}$, the starting point of the lemniscal pathway, better encoded under high-intensity background, whereas SpVi neurons, the origin of the paralemniscal pathway, efficiently encode during periods of weak tactile stimulation. Furthermore, these differences are already observable at the level of the subthreshold synaptic potentials.

Adaptation due to vibrissa stimulation near whisking frequency was previously reported in the two brainstem nuclei that we recorded from in this study (Jacquin et al., 1986; Deschênes et al., 2003; Minnery and Simons, 2003). It is believed that adaptation in this system arises from short-term synaptic depression (Tsodyks and Markram, 1997; Chung et al., 2002). This mecha- nism predicts more adaptation when stimulus intensity is increased, as it should increase presynaptic firing probability and consequently leads to higher utilization of synaptic resources. However, our previous study shows that in contrast to this prediction and throughout the lemniscal pathway, starting from the output of the brainstem PrV nucleus and up to cortex, increasing the intensity of stimulation entails less adaptation (Ganmor et al., 2010). More recently, we showed that the synaptic input to the PrV cells also adapts less as intensity increased (Mohar et al., 2013), strongly suggesting that short-term synaptic depression alone is unlikely to explain this adaptation profile, and may additionally require network or intrinsic mechanisms. In contrast, the synaptic adaptation profiles in the SpVi (the starting point of the paralemniscal pathway) followed the predictions of the shortterm synaptic depression model, as more adaptation was found as intensity increased. This assumption is further supported by the similarity of the unadapted and the adapted response curves of SpVi cells at the range of the low-intensity context (Fig. 3F, black and blue circles, respectively).

We hypothesize that these opposite adaptation patterns partially explain our results. Although the unadapted intensityresponse curves for both nuclei saturated already at low velocities, the adapted curves are very distinct from each other, demonstrating optimal coding at different intensity ranges where adaptation effects are lower (compare Fig. $3 E, F$ and Mohar et al., 2013, their Fig. 2). Linearization of the response curve for the high intensity in PrV cells is reminiscent of the effect of adapta- 
tion in the barrel cortex (Adibi et al., 2013), suggesting that this adaptation effect cascades all the way to the barrel cortex. Yet, the exact mechanisms that are responsible for the linearization of $\mathrm{PrV}$ response at high-intensity context or to saturation of SpVi response remains to be studied. Because our study was conducted using punctate stimuli, we note that additional research is required to generalize our conclusions to other types of tactile stimuli. Whether or not the specialization of these two pathways in encoding different intensities is related to their proposed roles in providing touch and whisking signals (Yu et al., 2006), or tactile versus noxious inputs (Sewards and Sewards, 2002) remains to be studied.

Similar to the visual and auditory systems, where low and high temporal and spatial acuity signals are conveyed by different pathways (Shapley et al., 1981; Calford and Aitkin, 1983), our data indicate that lemniscal cells in the brainstem are optimized for coding high-intensity context of stimuli (Wolfe et al., 2008), such as encountered when animals sweep their whiskers at high speed against textures, whereas paralemniscal cells encode subtle details, such as those expected during fine palpation.

In summary, we showed that adaptation in two major subcortical nuclei expands the range of intensity coding in the somatosensory system, potentially allowing the system to faithfully represent the instantaneous intensity of stimulation at different contexts.

\section{Notes}

Supplemental material for this article is available at www.weizmann.ac. il/neurobiology/labs/lampl/pdf/Mohar2015Supp.pdf. This document contains one supplemental figure, supplemental methods, and references. This material has not been peer reviewed.

\section{References}

Adibi M, McDonald JS, Clifford CW, Arabzadeh E (2013) Adaptation improves neural coding efficiency despite increasing correlations in variability. J Neurosci 33:2108-2120. CrossRef Medline

Averbeck BB, Latham PE, Pouget A (2006) Neural correlations, population coding and computation. Nat Rev Neurosci 7:358-366. CrossRef Medline

Barlow HB, Levick WR (1969) Changes in the maintained discharge with adaptation level in the cat retina. J Physiol 202:699-718. CrossRef Medline

Calford MB, Aitkin LM (1983) Ascending projections to the medial geniculate body of the cat: evidence for multiple, parallel auditory pathways through thalamus. J Neurosci 3:2365-2380. Medline

Chatterjee S, Callaway EM (2003) Parallel colour-opponent pathways to primary visual cortex. Nature 426:668-671. CrossRef Medline

Chung S, Li X, Nelson SB (2002) Short-term depression at thalamocortical synapses contributes to rapid adaptation of cortical sensory responses in vivo. Neuron 34:437-446. CrossRef Medline

Cohen-Kashi Malina K, Jubran M, Katz Y, Lampl I (2013) Imbalance between excitation and inhibition in the somatosensory cortex produces postadaptation facilitation. J Neurosci 33:8463-8471. CrossRef Medline

Dahmen JC, Keating P, Nodal FR, Schulz AL, King AJ (2010) Adaptation to stimulus statistics in the perception and neural representation of auditory space. Neuron 66:937-948. CrossRef Medline

Deschênes M, Timofeeva E, Lavallée P (2003) The relay of high-frequency sensory signals in the whisker-to-barreloid pathway. J Neurosci 23:6778 6787. Medline

Diamond ME, Armstrong-James M, Ebner FF (1992) Somatic sensory responses in the rostral sector of the posterior group (POm) and in the ventral posterior medial nucleus (VPM) of the rat thalamus. J Comp Neurol 318:462-476. CrossRef Medline

Fairhall AL, Lewen GD, Bialek W, de Ruyter Van Steveninck RR (2001) Efficiency and ambiguity in an adaptive neural code. Nature 412:787-792. CrossRef Medline

Furuta T, Urbain N, Kaneko T, Deschênes M (2010) Corticofugal control of vibrissa-sensitive neurons in the interpolaris nucleus of the trigeminal complex. J Neurosci 30:1832-1838. CrossRef Medline

Ganmor E, Katz Y, Lampl I (2010) Intensity-dependent adaptation of cortical and thalamic neurons is controlled by brainstem circuits of the sensory pathway. Neuron 66:273-286. CrossRef Medline

Jacquin MF, Mooney RD, Rhoades RW (1986) Morphology, response properties, and collateral projections of trigeminothalamic neurons in brainstem subnucleus interpolaris of rat. Exp Brain Res 61:457-468. Medline

Katz Y, Heiss JE, Lampl I (2006) Cross-whisker adaptation of neurons in the rat barrel cortex. J Neurosci 26:13363-13372. CrossRef Medline

Minnery BS, Simons DJ (2003) Response properties of whisker-associated trigeminothalamic neurons in rat nucleus principalis. J Neurophysiol 89: 40-56. CrossRef Medline

Mohar B, Katz Y, Lampl I (2013) Opposite adaptive processing of stimulus intensity in two major nuclei of the somatosensory brainstem. J Neurosci 33:15394-15400. CrossRef Medline

Poulet JF, Fernandez LM, Crochet S, Petersen CC (2012) Thalamic control of cortical states. Nat Neurosci 15:370-372. CrossRef Medline

Quiroga RQ, Nadasdy Z, Ben-Shaul Y (2004) Unsupervised spike detection and sorting with wavelets and superparamagnetic clustering. Neural Comput 16:1661-1687. CrossRef Medline

Sewards TV, Sewards M (2002) Separate, parallel sensory and hedonic pathways in the mammalian somatosensory system. Brain Res Bull 58:243260. CrossRef Medline

Shapley R, Kaplan E, Soodak R (1981) Spatial summation and contrast sensitivity of $\mathrm{X}$ and $\mathrm{Y}$ cells in the lateral geniculate nucleus of the macaque. Nature 292:543-545. CrossRef Medline

Simoncelli EP, Olshausen BA (2001) Natural image statistics and neural representation. Annu Rev Neurosci 24:1193-1216. CrossRef Medline

Tsodyks MV, Markram H (1997) The neural code between neocortical pyramidal neurons depends on neurotransmitter release probability. Proc Natl Acad Sci U S A 94:719-723. CrossRef Medline

Wark B, Lundstrom BN, Fairhall A (2007) Sensory adaptation. Curr Opin Neurobiol 17:423-429. CrossRef Medline

Wolfe J, Hill DN, Pahlavan S, Drew PJ, Kleinfeld D, Feldman DE (2008) Texture coding in the rat whisker system: slip-stick versus differential resonance. PLoS Biol 6:e215. CrossRef Medline

Yu C, Derdikman D, Haidarliu S, Ahissar E (2006) Parallel thalamic pathways for whisking and touch signals in the rat. PLoS Biol 4:e124. CrossRef Medline

Zagha E, Casale AE, Sachdev RN, McGinley MJ, McCormick DA (2013) Motor cortex feedback influences sensory processing by modulating network state. Neuron 79:567-578. CrossRef Medline 